\title{
INSTANTANEOUS ANGULAR SPEED: ENCODER-COUNTER ESTIMATION COMPARED WITH VIBRATION DATA
}

\author{
Marco SPAGNOL ${ }^{1}$, Luigi BREGANT ${ }^{2}$ \\ ${ }^{1}$ Università degli Studi di Trieste, via A. Valerio 10, 34127 Trieste TS Italy \\ mspagnol@units.it \\ ${ }^{2}$ Università degli Studi di Trieste, via A. Valerio 10, 34127 Trieste TS Italy \\ bregant@units.it
}

\begin{abstract}
In rotating machinery, actions of the moving parts take place at specific angular positions rather than at specific times. For this reason, having a geometrical reference, such as the one provided by an encoder, and studying the Instantaneous Angular Speed (IAS) variations can provide a large amount of information about the health status of the machine. In fact, from the variation of the IAS during the machine loads' cycle it is possible to identify defects and faults. The current work focuses on the estimation of the IAS through the Elapsed Time (ET) method, using a counter in order to measure the time elapsed between the pulses of an encoder. Both IAS and vibration measurement are conducted on an asynchronous four poles electrical motor driven by $50 \mathrm{~Hz}$ line current, without load. The study compares the order analysis of both signals. The bearing's Fundamental Train Frequency is detected using IAS estimation.
\end{abstract}

Keywords: instantaneous angular speed, encoder, elapsed time method, AC DC electrical machine, slip effect.

\section{ENCODER-COUNTER SYSTEM}

Among the different processing strategies to obtain IAS [Li et al - 2005], in this study, the Elapsed Time (ET) method is used. In this case, the counter measures the time elapsed between two successive pulses of the encoder. With this approach there are as many measurement values as there are pulses/revolution of the encoder. The frequency of the counter and the number of pulses determine the resolution of the IAS estimates. The method is strictly correlated to the real rotational angle of the shaft, except from the encoder tolerances. [André, et al - 2012], [Youssef, et al 2011], [Renaudin, et al - 2010]. A National Instruments $80 \mathrm{MHz}$ counter is used for the measurements described in the following paragraphs. The selected board allows a choice between three counting methods: the Elapsed Time (ET) method, using one counter, and the HighFrequency (HF) and Large-Range (LR) methods, using two counters. A comparison between these methods is reported in [Spagnol, et al - 2013]. In this paper ET method is used: the rising edge of the input signal of the encoder triggers the counting of the timebase ticks (Fig. 1). Since the timebase is of a known frequency, the frequency of the input signal can be obtained as (3).

$$
\begin{gathered}
f_{\text {count }}=80 \mathrm{MHz} \\
\Delta t_{\text {count }}=f_{\text {count }}^{-1}=1,25 \times 10^{-8} \mathrm{~s} \\
f_{\text {input }}=\frac{1}{n_{\text {count }} \Delta t_{\text {count }}}
\end{gathered}
$$

IAS measurement errors come from different sources. Generally speaking, the absolute error value, increases linearly with the speed and the resolution of the encoder, considering that the upper measured speed limit is the ratio between the encoder's resolution and the clock frequency of the counter.

The ideal encoder assumes exactly equal geometric segments and any variation causes the ET to be sampled on a non-uniform angular basis. Since the spacing pattern repeats itself after each revolution, the error manifests itself as high-level content at integer multiples of the shaft running speed. It is possible to use the synchronous averaged encoder passage times to correct for the uneven encoder spacing [Resor, et al - 2005]. These errors are unavoidable, but the production standards are very high and great precision can be obtained.

The ET measurement depends on the achievable time resolution, governed by the clock rate and the zero crossing detection circuit. These lead to two main problems: the counting method and the clock stability. Different authors [Youssef, et al - 2011], [André, et al - 2012] have analysed the problems and have suggested appropriate remedies.

Further errors can be experienced if the sensor undergoes lateral movement, if it is installed with eccentricity or misalignment, or if any light-path transmission variations are present, as shown from the test setup (Fig. 2). 
Period

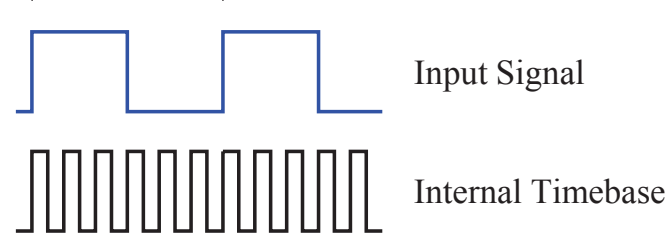

Fig. 1. Digital signal frequency measured with ET method

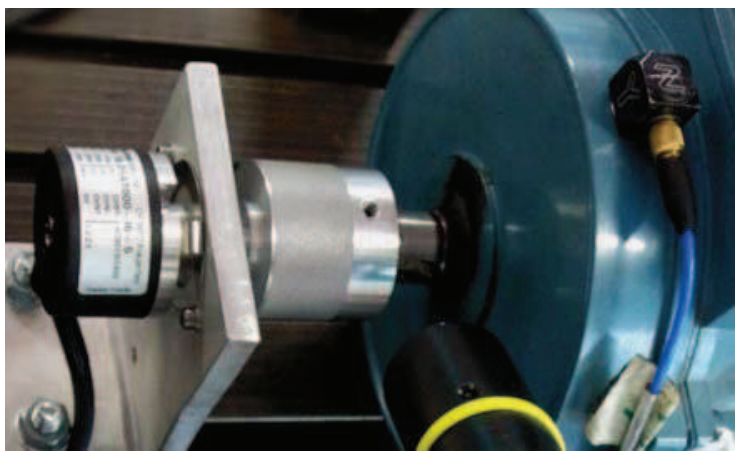

Fig. 2. Test setup: encoder 1000ppr, accelerometer $\mathrm{PCB}, 1 \mathrm{~kW} 4$ poles induction motor

\section{EXPERIMENTAL RESULTS}

In this paper, the IAS is compared to the vibration measurement obtained from accelerometer data. The motor used for the test is a $1 \mathrm{~kW} 4$ poles electric induction motor. It is driven by $50 \mathrm{~Hz}$ line current $380 \mathrm{~V}$. The test was conducted without load in order to emphasize speed variations. The encoder has 1000 pulse per revolution (ppr) and its shaft is rigidly connected to the motor's one. The encoder's body is fixed at the baseplate through a spring. The accelerometer is a PCB 356A16 with the $\mathrm{X}$ axis in tangential, the $\mathrm{Y}$ in the axial and the $Z$ in the radial direction. The acquisition system is based on National Instruments hardware. Analog signals are collected at $51.2 \mathrm{kHz}$, while the encoder's signal uses a $80 \mathrm{MHz}$ counter. A MATLAB software does the necessary signal processing. Due to the motor type, the slip effect is present. It is possible to view this phenomenon in Fig. 3, where signals from an AC and a DC motor are compared. The main component of the signal is the electro-magnetic force seen by the rotor and its frequency is given by (4), where $p$ is the number of poles, $f_{m}$ is the mechanical rotational frequency and $f_{e}$ is the electrical rotational frequency. In time/angular-domain, it appears as a shifting waveform due to the fact that there is a difference between the rotating speed to the magnetic field and the rotor. This effect doesn't appear with synchronous or DC machines because mechanical and electrical speeds are synchronized.

$$
f_{e}=\frac{f_{m}}{1-s} \frac{p}{2} \Rightarrow o_{e}=\frac{1}{1-s} \frac{p}{2}
$$

a)
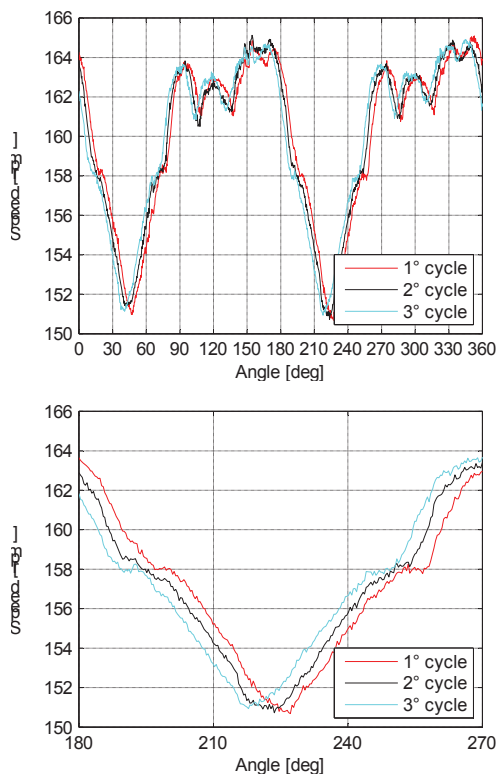

b)
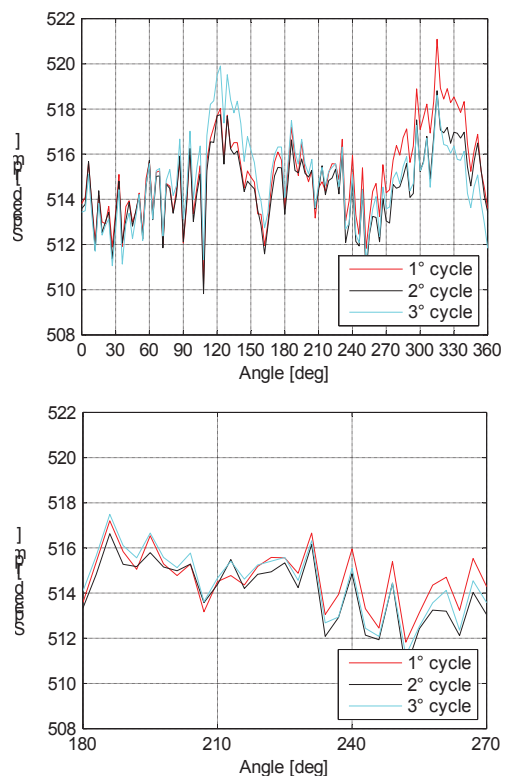

Fig. 3 . Three cycles at $0-360^{\circ}$ and zoom at $180-$ $270^{\circ}$ : a) AC motor, $150 \mathrm{rpm}$, encoder $1000 \mathrm{ppr}$, with slip effect; b) DC motor, 515rpm, encoder 120ppr, without slip effect

Table 1. Characteristic orders, bearing 6204-2RS1

\begin{tabular}{|c|c|c|}
\hline Defect & & Order \\
\hline $\begin{array}{l}\text { Bass Pass Frequency - } \\
\text { Inner }\end{array}$ & BPFI & 4.948 \\
\hline $\begin{array}{l}\text { Bass Pass Frequency - } \\
\text { Outer }\end{array}$ & BPFO & 3.052 \\
\hline $\begin{array}{ll}\text { Fundamental } & \text { Train } \\
\text { Frequency (Cage) } & \end{array}$ & FTF & 0.382 \\
\hline $\begin{array}{l}\text { Ball Spin Frequency } \\
\text { (Rolling element) }\end{array}$ & $\mathrm{BSF}$ & 1.992 \\
\hline
\end{tabular}


The motor under test has a working history. The measurements present electric phase imbalance since the fourth order is dominant. The characteristic frequencies of the installed bearing (type 6204-2RS1) are listed in Table 1.

In order to compare the measurements from the encoder and the accelerometer, an order analysis is performed. To visualize the results the order domain is chosen. The encoder signal is already in the proper domain, while the accelerometer output needs an angular resampling. The comparison presents the IAS orders obtained with the ET of the $1000 \mathrm{ppr}$ encoder signal and the accelerometer signals resampled at $1000 \mathrm{ppr}$. The encoder signal shows that at low order, the signal is good, while at higher order there is some quantization error, [André, et al - 2012]. The higher the speed, the bigger the quantization effect.

The order analysis from IAS and accelerometer data presents similar sidebands in the low order region. $f_{m}$ is the mechanical frequency, $f_{e}$ is the electrical supply frequency, $f_{s}$ is the synchronous frequency and depends on the number of poles. The motor actual speed is $1497 \mathrm{rpm}$ while the theoretical is $1500 \mathrm{rpm}$. The slip (6) between the two is 0.002 , that appears in the orders plot.

$$
\begin{gathered}
f_{s}=\frac{2 f_{e}}{p}=\frac{2}{4} 50 \mathrm{~Hz}=25 \mathrm{~Hz} \\
s=\frac{f_{s}-f_{m}}{f_{s}}=\frac{25-24.95}{25}=0.002
\end{gathered}
$$

It is present an order 0.008 due to the slip and pole pass. In this case there arefour poles, so pps is obtained in (7).

$$
\text { pps }=p \cdot s=4 \cdot 0.002=0.008
$$

Due to (4), the phase imbalance is present at $2 o_{e}$, obtaining order 4.008, (9).

$o_{e}=\frac{1}{1-s} \frac{p}{2}=\frac{1}{1-0.002} \frac{4}{2}=2.004$

$2 o_{e}=2 \cdot 2.004=4.008$

$4 o_{e}=4 \cdot 2.004=8.016$

$6 o_{e}=6 \cdot 2.004=12.024$

Figure 4 presents four plots around orders 2, 4, 8 and 12 . Orders at slip frequency harmonics are shown. The 4.008 order has the highest amplitude. There are sidebands at 0.008 around each peak.
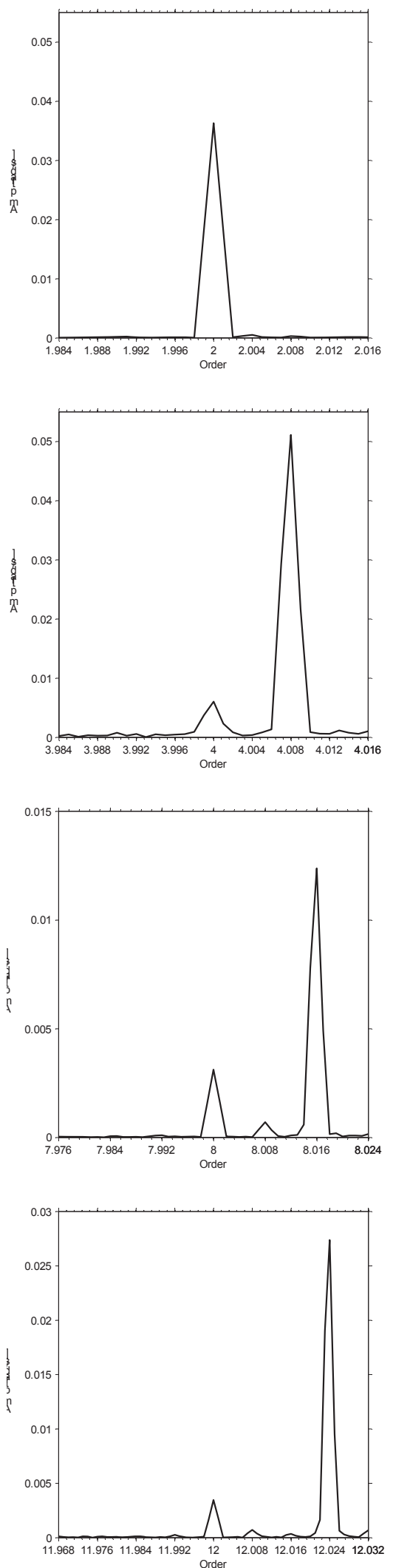

Fig. 4. IAS: slip order 2.004 and its harmonics $4.008,8.016$ and 12.024 
Figures 5-7 show IAS plots from encoder's data in the first subplot, while in the following three, acceleration's data in the three directions are reported. Figure 5a presents the measurement from 0 to the maximum order (500), while Fig. $5 \mathrm{~b}$ zooms from 0 to the 10 order. Encoder's main orders are 1, 2, 4.008, while acceleration's main orders are 1, 4.008 and 8.016. Figure 6 zooms around the fourth order where the 4.008 order appears in all signals. All the peaks are surrounded by sidebands at 0.008 . These are more evident at higher orders, especially in accelerometer data. IAS also shows sidebands at FTF order 0.382. The same sidebands are present in accelerometer data, but at higher orders and the peaks are smeared. Other sidebands are present at order 0.064 . These are related to the number of rotor bars, 32. Figure 7 zooms around the 8 order. The 8.016 order is shown and sidebands at 0.008 are present. The sidebands at 0.064 are not symmetrical.

a)

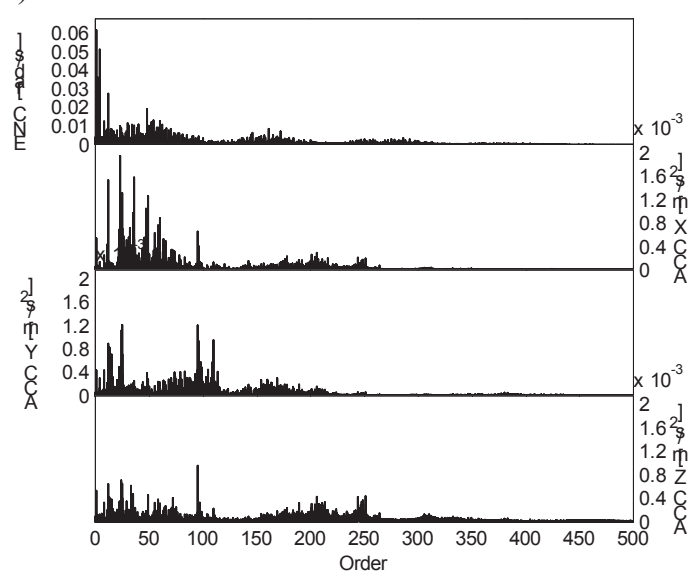

b)

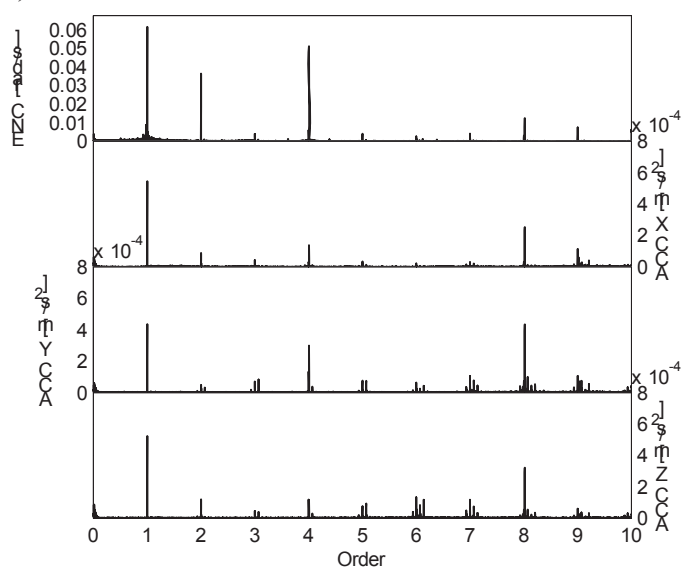

Fig. 5. IAS and accelerometer order analysis. The first subplot represents the encoder's data, while the three under are the accelerometer's data in $\mathrm{X}, \mathrm{Y}$, and $\mathrm{Z}$ directions. a) full scale plot, 0-500 order; b) zoom plot $0-10$ order a)

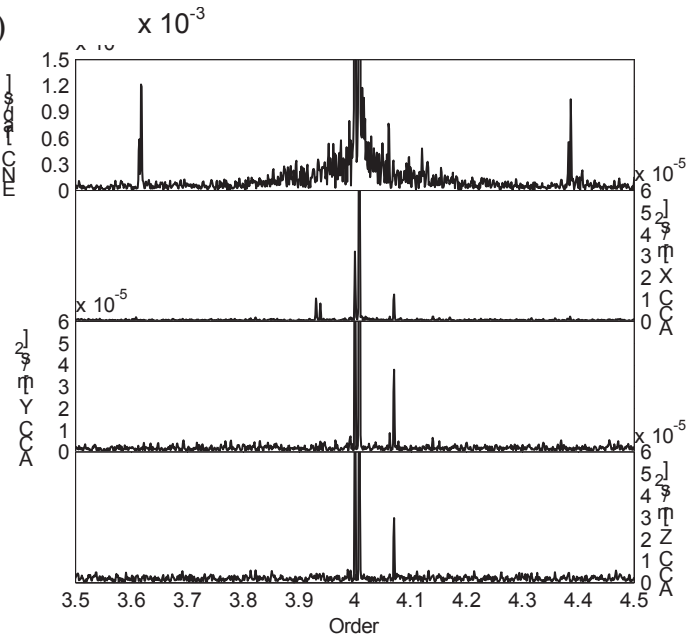

b)

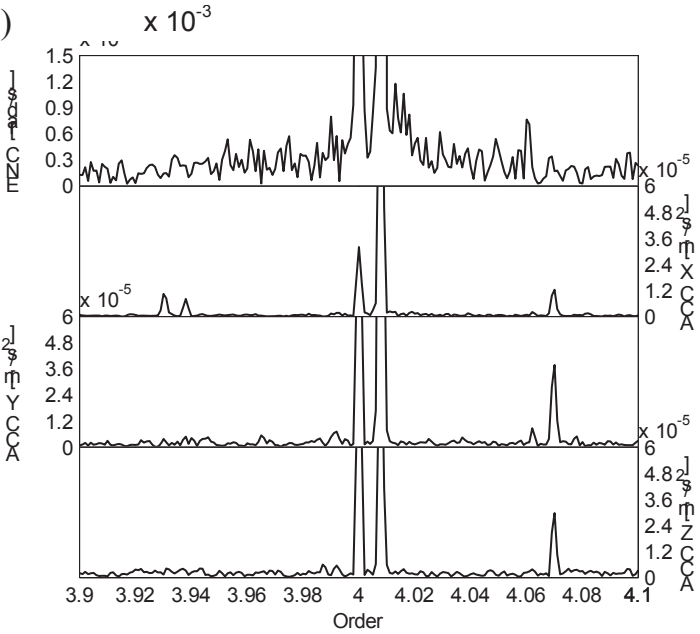

Fig. 6. IAS and accelerometer order analysis. The first subplot represents the encoder's data, while the three under are the accelerometer's data in X,Y, and $\mathrm{Z}$ directions. a) zoom plot, 3.5-4.5 order; b) zoom plot 3,9-4,1 order 
a)

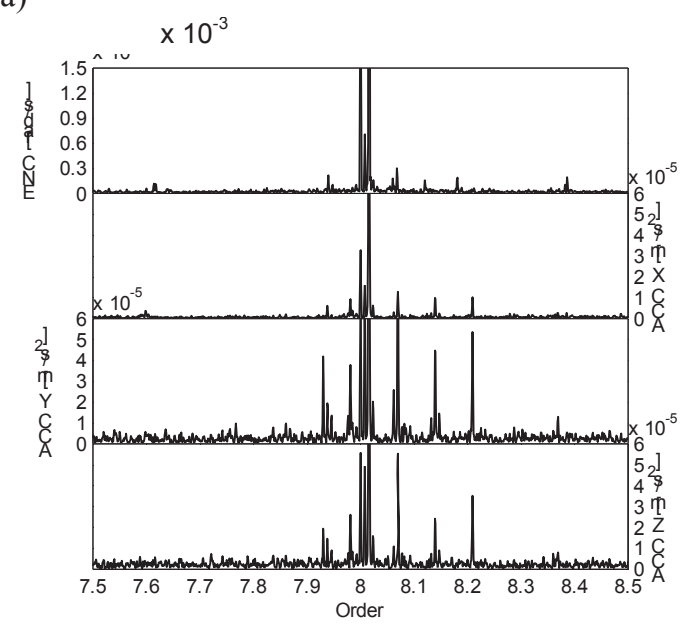

b)

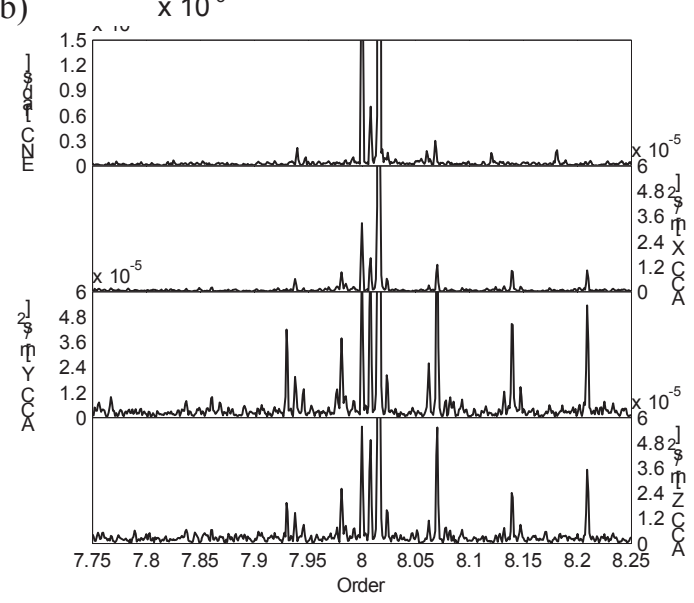

Fig. 7. IAS and accelerometer order analysis. The first subplot represents the encoder's data, while the three under are the accelerometer's data in $\mathrm{X}, \mathrm{Y}$, and $\mathrm{Z}$ directions. a) zoom plot, 7.5-8.5 order; b) zoom plot 7.75-8.25 order

\section{CONCLUSIONS}

This research shows the capability of IAS to identify FTF bearing defect. The electrical behaviour is the principal element of IAS order analysis, so the method can be used for diagnosis for electrical and mechanical defects. Further analysis has to be done to improve the signal processing technique but the comparison between IAS and acceleration measurements showed interesting differences.

\section{Acknowledgements}

This research is supported by Università degli Studi di Trieste, Nidec ASI S.p.A. and "Programma Operativo del Fondo Sociale Europeo 2007/2013 della Regione Autonoma Friuli Venezia Giulia".

\section{References}

[1] André, H. Bourdon, A. Antoni, J. Remond, D. (2012). Precision of the IAS monitoring system based on the elapsed time method in the spectral domain. Proceedings of the International Conference on Noise and Vibration Engineering ISMA 2012, Belgium, pp. 1126-1142.

[2] Li, Y. Gu, F. Harris, G. Ball, A.D. Bennett, N. Travis, K. (2005). The measurement of instantaneous angular speed. Mechanical Systems and Signal Processing, Vol. 19, no. 4, pp. 786-805.

[3] National Instruments (2013). Frequency Measurements: How-To Guide. Tutorial 7111

[4] Renaudin, L. Bonnardot, F. Musy, O. Doray, JB. Remond, D. (2010) Natural roller bearing fault detection by angular measurement of true instantaneous angular speed, Mechanical Systems and Signal Processing, Vol. 24, no. 7, pp. 1998-2011.

[5] Resor, BR. Trethewey, MMW. Maynard, KPK. (2005). Compensation for encoder geometry and shaft speed variation in time interval torsional vibration measurement. Journal of Sound and Vibration, Vol. 286, no. 4-5, pp. 897-920.

[6] Spagnol, M. Bregant, L. Boscarol, A. (2013) Instantaneous Angular Speed: comparisons between Torsional Laser Vibrometer and Encoder-Counter estimations. Proceedings of COMADEM2013.

[7] Youssef, W. Guillet, F. Elbadaoui, M. (2011) Characterization of counter technique for instantaneous angular speed measurement: Application on gear box signal. Proc. of the ICS6, Université Technologique de Compiègne, France. 